\title{
Predicting the superpartner spectrum from partially composite supersymmetry
}

\author{
Yusuf Buyukdag, ${ }^{*}$ Tony Gherghetta, ${ }^{\dagger}$ and Andrew S. Miller \\ School of Physics and Astronomy, University of Minnesota, Minneapolis, Minnesota 55455, USA
}

(Received 27 November 2018; published 15 March 2019)

\begin{abstract}
We use the idea of partial compositeness in a minimal supersymmetric model to relate the fermion and sfermion masses. By assuming that the Higgs and third-generation matter is (mostly) elementary, while the first- and second-generation matter is (mostly) composite, the Yukawa coupling hierarchy can be explained by a linear mixing between elementary states and composite operators with large anomalous dimensions. If the composite sector also breaks supersymmetry, then composite sfermions such as selectrons are predicted to be much heavier than the lighter elementary stops. This inverted sfermion mass hierarchy is consistent with current experimental limits that prefer light stops $(\mathcal{O}(10) \mathrm{TeV})$ to accommodate the $125 \mathrm{GeV}$ Higgs boson, while predicting heavy first- and second-generation sfermions $(\gtrsim 100 \mathrm{TeV})$ as indicated by flavor physics experiments. The underlying dynamics can be modeled by a dual $5 \mathrm{D}$ gravity theory that also predicts a gravitino dark matter candidate $(\gtrsim \mathrm{keV})$, together with gauginos and Higgsinos, ranging from $10-90 \mathrm{TeV}$, that are split from the heavier first- and second-generation sfermion spectrum. This intricate connection between the fermion and sfermion mass spectrum can be tested at future experiments.
\end{abstract}

DOI: $10.1103 /$ PhysRevD.99.055018

\section{INTRODUCTION}

Supersymmetry provides a compelling theoretical framework for addressing some of the shortcomings of the Standard Model of particle physics. These include dark matter, gauge coupling unification, and the stabilization of the hierarchy between the electroweak and Planck scales. Because supersymmetry must be broken, the stability of the electroweak scale requires that the sparticle spectrum should not be too heavy. A vital clue for determining the superpartner mass scale comes from the recent discovery of the $125 \mathrm{GeV}$ Higgs boson [1,2]. To obtain this mass in minimal supersymmetry, the Higgs quartic coupling must receive sizeable radiative corrections. These can arise from the top quark superpartners (or stops), provided that the lightest stop has mass of $\mathcal{O}(10) \mathrm{TeV}$. In the minimal framework there are no other sizeable contributions to the Higgs quartic coupling, and consequently the rest of the sparticle spectrum is not determined. The spectrum must only be compatible with the current LHC limits that require stop masses to be $\gtrsim 1120 \mathrm{GeV}$ [3] and gluino masses $\gtrsim 1970 \mathrm{GeV}$ [4].

\footnotetext{
*buyuk007@umn.edu

†tgher@umn.edu

mill5738@umn.edu
}

Published by the American Physical Society under the terms of the Creative Commons Attribution 4.0 International license. Further distribution of this work must maintain attribution to the author(s) and the published article's title, journal citation, and DOI. Funded by SCOAP.
Other indirect constraints such as the absence of flavorchanging processes, prefer the first- and second-generation scalar masses to be much heavier, $\gtrsim 100 \mathrm{TeV}$. Thus, the current experimental situation seems to suggest a sizeable hierarchy in the sfermion mass spectrum, which is inverted compared to the well-known fermion mass hierarchy. For instance, the electron (top quark) is the lightest (heaviest) charged fermion, while the selectron (stop) may be the heaviest (lightest) charged sfermion.

In this paper, we provide a mechanism that explains the origin of the inverted sfermion mass hierarchy and predicts the sparticle spectrum. The mechanism relies on partial compositeness [5], whereby the Standard Model fields are admixtures arising from the linear mixing of elementary states with composite operators. Assuming that the Higgs fields are elementary, the magnitudes of the corresponding Yukawa couplings then depend on the relative compositeness of the Standard Model fermions. To obtain an orderone Yukawa coupling with the Higgs, the top quark must be mostly elementary, while, since the elementary and composite sectors mix with an irrelevant coupling, the smallness of the electron Yukawa coupling follows from assuming that the electron is mostly composite. The remainder of the Standard Model Yukawa couplings are generated by varying degrees of compositeness. If one now further supposes that the composite sector is responsible for breaking supersymmetry, then an inverted hierarchy immediately follows. Selectrons, which are mostly composite, receive large supersymmetry-breaking masses, while stops, which are elementary, obtain suppressed 
supersymmetry-breaking contributions. In this way the fermion mass hierarchy determines the sfermion mass hierarchy and predicts an inverted mass spectrum.

The underlying strong dynamics that would be responsible for such a mechanism is similar to single-sector models of supersymmetry breaking that were originally proposed in [6,7], with related work in [8-12]. Even if the underlying gauge theory were completely known, however, predictions for the spectrum would be difficult to obtain due to the nonperturbative dynamics. Therefore, we will instead use the AdS/CFT correspondence to model the strong dynamics with a slice of $\mathrm{AdS}_{5}$. In light of the Higgs boson discovery, this enables us to obtain specific quantitative predictions for the sparticle spectrum that can then be used to help guide future experimental searches.

\section{PARTIALLY COMPOSITE SUPERSYMMETRY}

To illustrate the mechanism of partially composite supersymmetry, consider the elementary chiral superfield $\Phi=\phi+\sqrt{2} \theta \psi+\theta \theta F$, where $\phi$ is a complex scalar, $\psi$ is a Weyl fermion, and $F$ is an auxiliary field. In addition, we introduce a corresponding supersymmetric chiral operator $\mathcal{O}=\mathcal{O}_{\phi}+\sqrt{2} \theta \mathcal{O}_{\psi}+\theta \theta \mathcal{O}_{F}$. The scaling dimensions of the component operators are $\operatorname{dim} \mathcal{O}_{\phi}=1+\delta_{\mathcal{O}}$, $\operatorname{dim} \mathcal{O}_{\psi}=\frac{3}{2}+\delta_{\mathcal{O}}$, and $\operatorname{dim} \mathcal{O}_{F}=2+\delta_{\mathcal{O}}$, where $\delta_{\mathcal{O}} \geq 0$ is the anomalous dimension [13].

The supersymmetric Lagrangian contains separate elementary and composite sectors, together with linear mixing terms of the form $\left[\Phi \mathcal{O}^{c}\right]_{F}$ for each chiral superfield $\Phi$ and charge-conjugate composite operator $\mathcal{O}^{c}$. At the UV scale $\Lambda_{\mathrm{UV}}$, it is given by

$$
\mathcal{L}_{\Phi}=\left[\Phi^{\dagger} \Phi\right]_{D}+\frac{1}{\Lambda_{\mathrm{UV}}^{\delta-1}}\left(\left[\Phi \mathcal{O}^{c}\right]_{F}+\text { H.c. }\right),
$$

where $\delta$ is the anomalous dimension of $\mathcal{O}^{c}$. We have taken order-one UV coefficients for the higher-dimension terms, and omitted a kinetic mixing between the elementary and composite sectors in our minimal setup. The composite sector is assumed to confine at an infrared scale, $\Lambda_{\mathrm{IR}}$. In the limit of large- $N$ strong dynamics, the two-point function for the composite operator components can be written as a sum over one-particle states. In particular, for the scalar component, the two-point function $\left\langle\mathcal{O}_{\phi}(p) \mathcal{O}_{\phi}(-p)\right\rangle=$ $\sum_{n} a_{n}^{2} /\left(p^{2}+m_{n}^{2}\right)$ to leading order in $1 / N$, where $a_{n}=$ $\left\langle 0\left|\mathcal{O}_{\phi}\right| n\right\rangle \propto \sqrt{N} /(4 \pi)$ is the matrix element for $\mathcal{O}_{\phi}$ to create the $n$th state with mass $m_{n}$ from the vacuum [14].

The elementary-composite mixing in the Lagrangian (1) mixes the elementary fields $(\phi, \psi)$ with the composite resonance states. Assuming for simplicity just the lowestlying composite state $\Phi^{(1)}=\left(\phi^{(1)}, \psi^{(1)}\right)$ with mass $g_{\Phi}^{(1)} \Lambda_{\mathrm{IR}}$, the two-state system can be diagonalized to obtain the massless eigenstate [15]

$$
\left|\Phi_{0}\right\rangle \simeq \mathcal{N}_{\Phi}\left\{|\Phi\rangle-\frac{1}{g_{\Phi}^{(1)} \sqrt{\zeta_{\Phi}}} \sqrt{\frac{\delta-1}{\left(\frac{\Lambda_{\mathrm{IR}}}{\Lambda_{\mathrm{UV}}}\right)^{2(1-\delta)}-1}}\left|\Phi^{(1)}\right\rangle\right\}
$$

where $\Phi_{0}=\left(\phi_{0}, \psi_{0}\right), g_{\Phi}^{(1)}$ and $\zeta_{\Phi}$ are order-one constants, and $\mathcal{N}_{\Phi}$ is a normalization constant. Given that $\Lambda_{\mathrm{IR}} \ll \Lambda_{\mathrm{UV}}$, this expression shows that the massless eigenstates are mostly elementary for $\delta>1$, whereas for $0 \leq \delta<1$ they are an admixture of elementary and composite states.

This elementary-composite admixture of the massless eigenstate can now be used to explain the fermion mass hierarchy [16], and then predict the sfermion mass spectrum. Consider elementary chiral fermions, $\psi_{L, R}$, that are coupled to the elementary Higgs field, $H$, via the Yukawa interaction $\lambda \psi_{L}^{\dagger} \psi_{R} H+$ H.c., where $\lambda$ is an order-one protoYukawa coupling (for simplicity, we assume one fermion generation and ignore the distinction between $H_{u}$ and $H_{d}$ ). Diagonalizing the fermion Lagrangian with the Higgs contribution gives the Yukawa coupling expression

$$
y_{\psi} \simeq \begin{cases}\frac{\lambda}{\zeta_{\Phi}}(\delta-1) \frac{16 \pi^{2}}{N} & \delta \geq 1, \\ \frac{\lambda}{\zeta_{\Phi}}(1-\delta) \frac{16 \pi^{2}}{N}\left(\frac{\Lambda_{\mathrm{IR}}}{\Lambda_{\mathrm{UV}}}\right)^{2(1-\delta)} & 0 \leq \delta<1,\end{cases}
$$

where we have assumed that $\delta \equiv \delta_{L}=\delta_{R}$. We clearly see that when $\delta \geq 1$ (corresponding to a mostly elementary fermion), the Yukawa coupling is of order one for sufficiently large $N$. Conversely, when $0 \leq \delta<1$ (corresponding to a sizeable composite admixture), the Yukawa coupling has a power-law suppression that depends on the degree of compositeness. This explains why composite fermions (identified with the first- and second-generation Standard Model fermions) have small Yukawa couplings, while elementary fermions (such as the top quark) have order-one Yukawa couplings.

The composite sector is also responsible for supersymmetry breaking. Soft scalar masses are generated only for the composite sector fields since there is no direct coupling of the supersymmetry breaking to elementary fields. For example, the massive scalar field, $\phi^{(1)}$ obtains a soft mass

$\xi_{4} \frac{g_{\Phi}^{(1) 2}}{\Lambda_{\mathrm{IR}}^{2}}\left[\mathcal{X}^{\dagger} \mathcal{X} \Phi^{(1) \dagger} \Phi^{(1)}\right]_{D}=\xi_{4} g_{\Phi}^{(1) 2} \frac{\left|F_{\mathcal{X}}\right|^{2}}{\Lambda_{\mathrm{IR}}^{2}} \phi^{(1) \dagger} \phi^{(1)}$,

where $\mathcal{X}=\theta \theta F_{\mathcal{X}}$ is a composite-sector spurion and $\xi_{4}$ is a dimensionless parameter. Given the scalar admixture (2), the corresponding sfermion mass-squared becomes:

$$
\tilde{m}^{2} \simeq \begin{cases}\frac{(\delta-1)}{\zeta_{\Phi}} \frac{16 \pi^{2}}{N} \frac{\left|F_{\mathcal{X}}\right|^{2}}{\Lambda_{\mathrm{IR}}^{2}}\left(\frac{\Lambda_{\mathrm{IR}}}{\Lambda_{\mathrm{UV}}}\right)^{2(\delta-1)} & \delta \geq 1, \\ \frac{(1-\delta)}{\zeta_{\Phi}} \frac{16 \pi^{2}}{N} \frac{\left|F_{\mathcal{X}}\right|^{2}}{\Lambda_{\mathrm{IR}}^{2}} & 0 \leq \delta<1,\end{cases}
$$

where, for a large- $N$ gauge theory, $\xi_{4} \simeq 16 \pi^{2} / N$ [14]. When the sfermion is mostly elementary $(\delta \geq 1)$, the soft mass is power-law suppressed since the supersymmetry 
breaking is transmitted via the elementary-composite mixing. (Note, however, that for sufficiently large $\delta$, radiative corrections will become increasingly important.) This contrasts with the case $0 \leq \delta<1$, where the mass eigenstate is mostly composite and there is no power-law suppression. Thus, elementary sfermions (identified with the stops) are much lighter than the composite sfermions (identified with the first- and second-generation sfermions), predicting an inverted mass hierarchy.

The fermion and sfermion mass hierarchies critically depend on the anomalous dimensions $\delta$. To illustrate this, we consider the $\delta_{e, t}$ values required to obtain the electron top-quark Yukawa coupling ratio $y_{e} / y_{t}$ at the IR scale. These are plotted in Fig. 1 for various values of $\Lambda_{\mathrm{IR}} / \Lambda_{\mathrm{UV}}$, where the ratio $y_{e} / y_{t}\left(\sim 10^{-5}\right)$ at the IR scale is determined via two-loop renormalization group evolution (assuming a universal soft mass threshold). Using (5), this then predicts the sfermion mass ratio $m_{\tilde{e}} / m_{\tilde{t}}$ at the IR scale. As shown in Fig. 1 , the allowed region is $0 \lesssim \delta_{e} \lesssim 0.9$ and $1 \lesssim \delta_{t} \lesssim 1.8$, depending on the value of $\Lambda_{\mathrm{IR}} / \Lambda_{\mathrm{UV}}$. The largest value of the ratio $m_{\tilde{e}} / m_{\tilde{t}}$ is approximately 140 (390) for $\Lambda_{\mathrm{IR}} / \Lambda_{\mathrm{UV}} \simeq 10^{-3}\left(10^{-16}\right)$. Note that the slanted horizontal contours in Fig. 1 end on the right, at the $\delta_{t}$ value for which radiative corrections to the soft mass (5) begin to dominate. These corrections are calculated in Ref. [15].

A partially composite analysis can also be done for the vector and gravity supermultiplets. They lead to a mostly

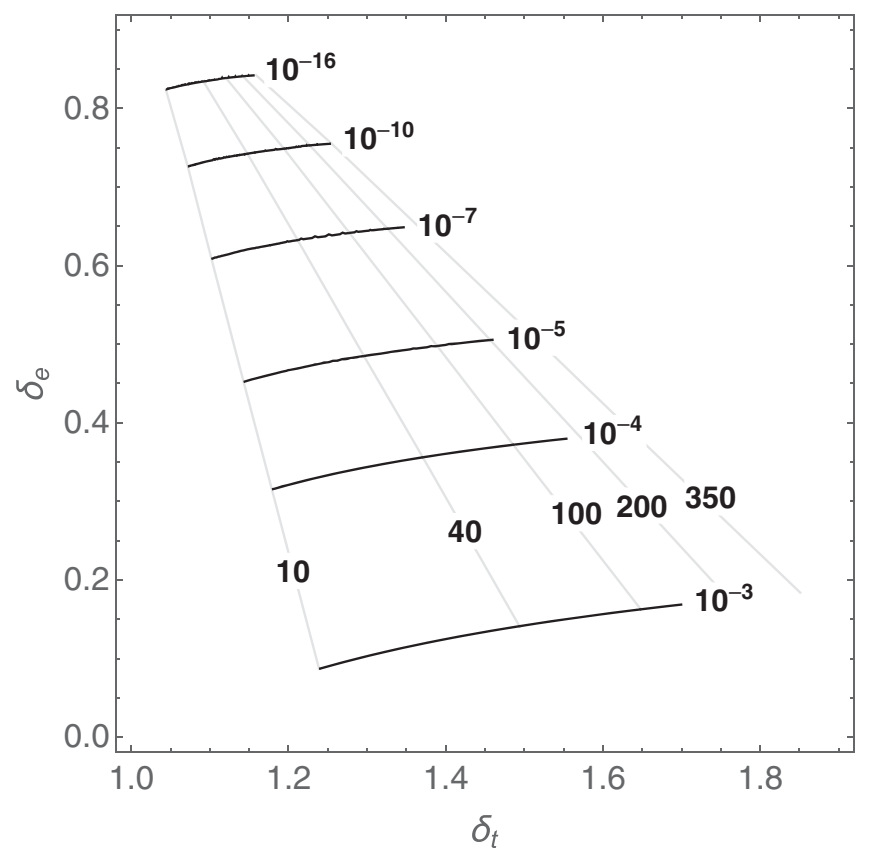

FIG. 1. The estimated range of anomalous dimensions $\delta_{e}, \delta_{t}$ that gives rise to the observed hierarchy between the electron and top quark Yukawa couplings, assuming $\Lambda_{\mathrm{UV}}=10^{18} \mathrm{GeV}$, $\tan \beta=3$, and a soft mass threshold at $50 \mathrm{TeV}$. The slanted horizontal and vertical lines are contours of the ratio $\Lambda_{\mathrm{IR}} / \Lambda_{\mathrm{UV}}$ and the sfermion mass ratio $m_{\tilde{e}} / m_{\tilde{t}}$, respectively. elementary gauge boson and gaugino, and an elementary graviton and gravitino [17]. Since supersymmetry breaking occurs in the composite sector, this implies that the gauginos are lighter than the mostly composite first- and secondgeneration sfermions and comparable in mass to the mostly elementary third-generation sfermions. On the other hand, since the gravitino has a tiny composite admixture, it is almost always the lightest supersymmetric particle (LSP). These are the qualitative features of the partially composite sparticle spectrum. Further details are presented in Ref. [15].

\section{A 5D GRAVITY MODEL}

The partially composite supersymmetric framework generically relates the fermion and sfermion mass spectra that result from some (unknown) strong dynamics. In order to model the underlying dynamics and obtain quantitative predictions, we now consider a five-dimensional (5D) dual gravity model that is motivated by the AdS/CFT correspondence [18]. The $5 \mathrm{D}$ spacetime, $\left(x^{\mu}, y\right)$, where $\mu=0,1$, 2, 3 labels the four-dimensional (4D) coordinates and the fifth coordinate, $y$, is compactified on an orbifold $\left(S^{1} / \mathbb{Z}_{2}\right)$. The anti-de Sitter (AdS) metric is given by

$$
d s^{2}=e^{-2 k y} d x^{2}+d y^{2},
$$

where $k$ is the AdS curvature scale. The 5D spacetime is a slice of $\mathrm{AdS}_{5}$ bounded by two 3-branes located at the orbifold fixed points: a UV brane at $y=0$ and an IR brane at $y=\pi R$, where $R$ is the orbifold radius [19].

Besides gravity, we introduce the full matter and gaugesector content of the minimal supersymmetric standard model in the $\mathrm{AdS}_{5}$ bulk. The $\mathcal{N}=1$ chiral matter and vector superfields are embedded into $5 \mathrm{D} \mathcal{N}=1$ hypermultiplets and vector supermultiplets, respectively. The 4D superfields are then identified with the massless zero modes, while the massive Kaluza-Klein states form $4 \mathrm{D} \mathcal{N}=2$ supermultiplets with masses of order $\Lambda_{\mathrm{IR}}$. The $\mathcal{N}=1$ Higgs supermultiplets, meanwhile, are 4D fields confined to the UV brane. In this setup, each fermion zero mode obtains a mass from a UV boundary-bulk Yukawa interaction with 5D Yukawa coupling, $Y^{(5)}$. The fermion mass hierarchy then arises from the overlap of the UV-localized Higgs fields with the left- and right-handed bulk fermion fields with profiles $\psi_{L, R} \propto e^{\left(\frac{1}{2} \mp c\right) k y}$ in the fifth dimension, where the $c$ are dimensionless bulk fermion mass parameters [20,21]. Once the $c$ parameters are determined for each fermion flavor, the sparticle mass spectrum can then be predicted.

Supersymmetry is only broken on the IR brane and can be parametrized by introducing a boundary interaction with a spurion $X=\theta^{2} F_{X}$ for each 5D hypermultiplet $\Phi\left(x^{\mu}, y\right)$ :

$$
\int d^{5} x \sqrt{-g} \int d^{4} \theta \frac{X^{\dagger} X}{\Lambda_{\mathrm{UV}}^{2} k} \Phi^{\dagger} \Phi \delta(y-\pi R) .
$$

This interaction leads to the sfermion soft mass 


$$
\tilde{m}_{L, R} \simeq \begin{cases}\left( \pm c-\frac{1}{2}\right)^{1 / 2} \frac{F}{\Lambda_{\mathrm{IR}}} e^{\left(\frac{1}{2} \mp c\right) \pi k R} & \pm c>\frac{1}{2} \\ \left(\frac{1}{2} \mp c\right)^{1 / 2} \frac{F}{\Lambda_{\mathrm{IR}}} & \pm c<\frac{1}{2}\end{cases}
$$

where $\sqrt{F} \equiv \sqrt{F_{X}} e^{-\pi k R}$, and the backreaction on the sfermion profile by the boundary mass is negligible (i.e., $\left.\sqrt{F} / \Lambda_{\mathrm{IR}} \lesssim 1\right)$. Furthermore, note that possible flavor offdiagonal mass terms in (7) have been neglected since the sfermion mass scale is assumed to be $\mathcal{O}(100) \mathrm{TeV}$. Using the AdS/CFT dictionary relations $\Lambda_{\mathrm{IR}} / \Lambda_{\mathrm{UV}}=e^{-\pi k R}$ and $\delta=\left|c \pm \frac{1}{2}\right|$, the expressions (8) are seen to be consistent with the masses (5) obtained in the 4D holographic theory. Quantum corrections to the tree-level scalar masses (8) arising from loops of bulk hypermultiplets and vector supermultiplets are important for suppressed masses $\left( \pm c \gtrsim \frac{1}{2}\right)$. These are computed in Ref. [15], and their effect is typically to reduce the sfermion mass hierarchy.

Similarly, introducing an IR-boundary gaugino interaction term $X W_{\alpha}^{a} W^{a \alpha}$, where $W_{\alpha}^{a}$ is the gauge field strength superfield, gives rise to gaugino masses $M_{\lambda_{a}} \simeq g_{a}^{2} F / \Lambda_{\mathrm{IR}}$ [22], with $g_{a}(a=1,2,3)$ the corresponding Standard Model gauge couplings. The gaugino masses are suppressed relative to the heavier sfermions (with $\pm c<\frac{1}{2}$ ). Alternatively, if the supersymmetry-breaking sector does not contain any gauge singlets, the gaugino masses may instead be generated by a boundary interaction $X^{\dagger} X W_{\alpha}^{a} W^{a \alpha}$. This leads to gaugino masses, $M_{\lambda_{a}} \simeq g_{a}^{2} F^{2} / \Lambda_{\mathrm{IR}}^{3}$ that are further suppressed.

When supersymmetry is spontaneously broken on the IR boundary, the effective $4 \mathrm{D}$ cosmological constant receives a positive contribution from $F_{X}$. In the 5D warped geometry, this contribution can be canceled by the addition of a constant superpotential $W$ on the UV brane [22-28], giving rise to a gravitino mass $m_{3 / 2} \simeq F /\left(\sqrt{3} M_{P}\right)$. Since the gravitational coupling is Planck-scale suppressed, the gravitino mass is lower than the characteristic soft-mass scale $F / \Lambda_{\mathrm{IR}}$ by a warp factor.

The Higgs sector does not couple directly to the IR brane, and therefore the Higgs soft terms $m_{H_{u}}^{2}, m_{H_{d}}^{2}$, and $b$ as well as the trilinear soft scalar couplings ( $a$-terms) at the IR-brane scale are zero at tree level. However, these soft terms are generated via radiative corrections from their interactions with bulk hypermultiplets and vector supermultiplets [15]. The resulting values for the Higgs soft masses, obtained at the IR-brane scale, must be run down to near the electroweak scale in order to check that electroweak symmetry is broken. The Higgs $\mu$-term is assumed to arise on the UV brane from a higher-dimensional superpotential term allowed by an extra $\mathrm{U}(1)$ symmetry, as in the Kim-Nilles mechanism [29]. Its value, along with $\tan \beta$ (the ratio of the Higgs vacuum expectation values), is determined by the conditions for electroweak symmetry breaking.
The parameters of the 5D model therefore consist of the IR brane scale, $\Lambda_{\mathrm{IR}}$, and the supersymmetry breaking scale, $\sqrt{F}$. In addition, there is a universal 5D Yukawa coupling, $Y^{(5)}$, and the six bulk fermion mass parameters $c_{L_{i}, Q_{i}}$ (one for each generation of leptons and quarks). These parameters can be used to determine both the fermion and sparticle mass spectra. However, there are a number of phenomenological and theoretical constraints which restrict the possible parameter values. These include:

(i) Gravitino dark matter: Assuming $R$-parity conservation, the gravitino LSP makes an excellent dark matter candidate, provided $m_{3 / 2} \gtrsim 1 \mathrm{keV}$ [30,31].

(ii) Higgs mass and electroweak symmetry breaking: The observed $125 \mathrm{GeV}$ Higgs boson can be accommodated if the mass of the lightest stop is $\mathcal{O}(10) \mathrm{TeV}$. Since the Higgs-sector soft terms are generated radiatively, the requirement that the Higgs scalar potential correctly breaks electroweak symmetry leads to further indirect constraints on the soft masses of the sfermions.

(iii) Supersymmetric flavor problem: To avoid generating excessive flavor-changing processes, the firstand second-generation sfermions must be at least $100 \mathrm{TeV}$.

(iv) Gauge coupling unification: To preserve the successful supersymmetric prediction of gauge coupling unification [assuming any underlying dynamics is $\mathrm{SU}(5)$ symmetric], the gaugino and Higgsino masses must be lighter than $300 \mathrm{TeV}$.

(v) Charge- and color-breaking minima: Since the predicted sfermion mass spectrum at $\Lambda_{\mathrm{IR}}$ is flavordependent and the first- and second-generation sfermions are typically hierarchically larger than the third-generation sfermions, there are both one-loop $D$-term and two-loop gauge contributions to scalar masses that can lead to charge and color-breaking minima.

Subject to the above constraints, we choose two benchmark scenarios corresponding to the singlet and nonsinglet spurion cases. The singlet case has parameter values $\Lambda_{\mathrm{IR}}=2 \times 10^{16} \mathrm{GeV}, \sqrt{F}=4.75 \times 10^{10} \mathrm{GeV}, Y^{(5)} k=1$, and $\tan \beta \simeq 3$ at the IR-brane scale, whereas the nonsinglet case has parameter values $\Lambda_{\mathrm{IR}}=6.5 \times 10^{6} \mathrm{GeV}$, $\sqrt{F}=2 \times 10^{6} \mathrm{GeV}, Y^{(5)} k=1$, and $\tan \beta \simeq 5$ at the IRbrane scale. The sfermion pole mass predictions are presented in Fig. 2, where the spread in the masses results from a scan over the $c$-parameters in order to fit the Yukawa coupling hierarchy. The Higgs mass lies in the range 124$126 \mathrm{GeV}$, with sign $\mu=-1$. Furthermore, the mass of the LSP gravitino is $535 \mathrm{GeV}(1 \mathrm{keV})$ for the singlet (nonsinglet) spurion case. The sfermion masses obtained directly result from explaining the fermion mass hierarchy. They reveal a distinctive, flavor-dependent inverted mass hierarchy, in contrast to usual supersymmetric models where scalar and gaugino masses are unconstrained by the fermion mass spectrum. 


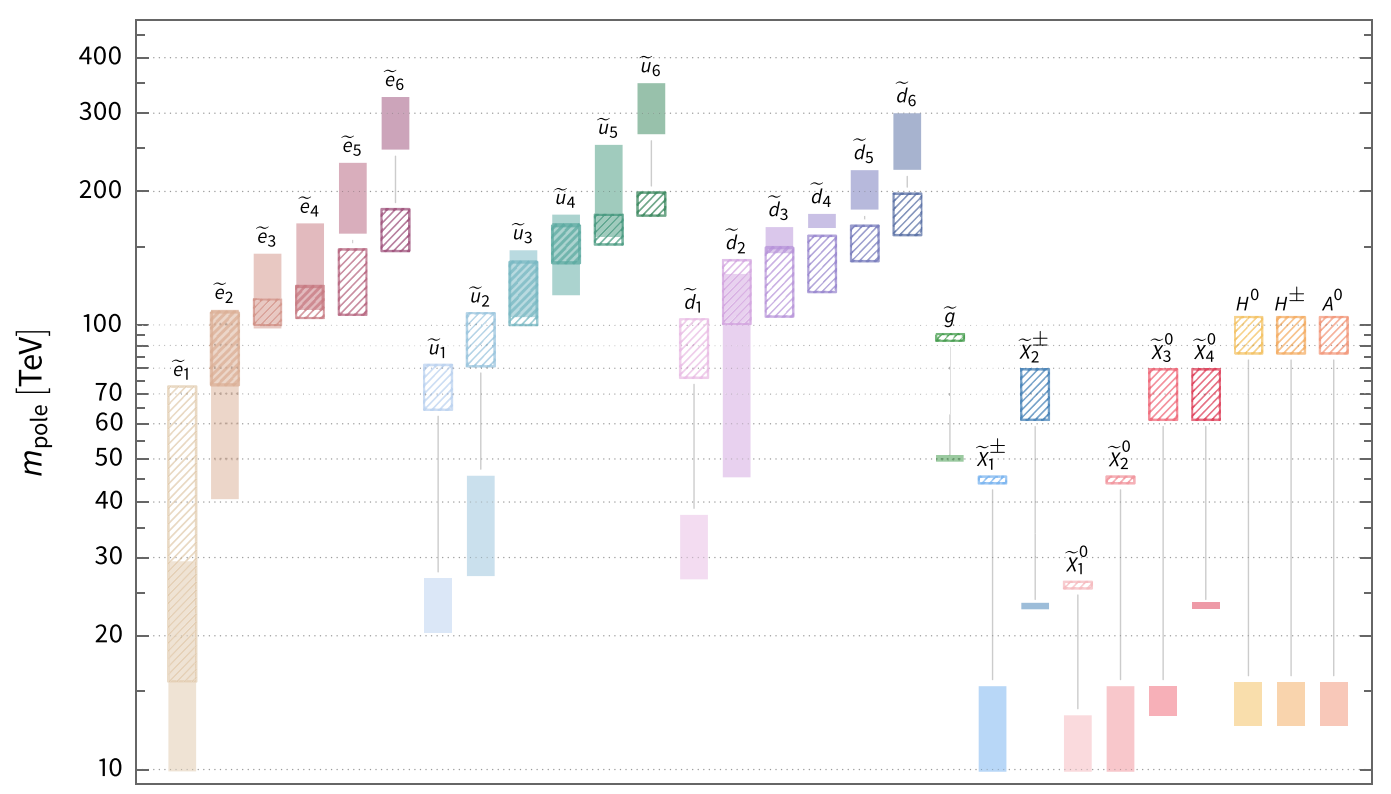

FIG. 2. The sparticle mass spectrum for two benchmark scenarios: singlet spurion case (hatched) with $\Lambda_{\mathrm{IR}}=2 \times 10^{16} \mathrm{GeV}$, $\sqrt{F}=4.75 \times 10^{10} \mathrm{GeV}, \tan \beta \sim 3$, and $Y^{(5)} k=1$; and nonsinglet spurion case (solid) with $\Lambda_{\mathrm{IR}}=6.5 \times 10^{6} \mathrm{GeV}, \sqrt{F}=2 \times 10^{6} \mathrm{GeV}$, $\tan \beta \sim 5$, and $Y^{(5)} k=1$.

\section{CONCLUSION}

In this paper, we have presented a partially composite supersymmetric model that assumes the first two generations of matter are (mostly) composite, while the Higgs and third generation matter are (mostly) elementary. This feature can then be used to explain the fermion mass hierarchy, predicting, as a consequence, a distinct sparticle mass spectrum with an inverted sfermion mass hierarchy: light stops and staus and heavy first-and second-generation sfermions. The underlying dynamics responsible for the compositeness can be modeled with a dual 5D gravity theory that further predicts a gravitino LSP, together with gauginos and Higgsinos ranging from the lightest neutralino at $10 \mathrm{TeV}$ to gluinos at $90 \mathrm{TeV}$. These masses are split from the heavier first- and secondgeneration sfermions, thereby preserving the successful supersymmetric prediction of gauge coupling unification. A more detailed analysis of this model is given in Ref. [15].

The partially composite supersymmetric model intricately connects the generation of the fermion mass hierarchy with the sfermion masses. It is striking that the predicted sparticle spectrum seems to provide an appealing fit to the current experimental constraints. While not directly accessible at the $13 \mathrm{TeV}$ LHC, the signatures of this sparticle spectrum, such as distinctive long-lived NLSP decays, may be within the reach of a future highenergy collider. Alternatively, the heavy first- and secondgeneration sfermions could be indirectly probed at flavorviolation experiments such as the Mu2e experiment [32] or at experiments aiming to measure the electric dipole moment of the electron [33]. Thus, partial compositeness and supersymmetry are intriguing possibilities that could together play a central role in addressing some of the shortcomings of the Standard Model.

\section{ACKNOWLEDGMENTS}

We thank Jason Evans, Ben Harling, and Alex Pomarol for helpful discussions. This work is supported in part by the U.S. Department of Energy under Grant No. DESC0011842 at the University of Minnesota.
[1] G. Aad et al. (ATLAS Collaboration), Observation of a new particle in the search for the Standard Model Higgs boson with the ATLAS detector at the LHC, Phys. Lett. B 716, 1 (2012).
[2] S. Chatrchyan et al. (CMS Collaboration), Observation of a new boson at a mass of $125 \mathrm{GeV}$ with the CMS experiment at the LHC, Phys. Lett. B 716, 30 (2012). 
[3] A. M. Sirunyan et al. (CMS Collaboration), Search for top squark pair production in pp collisions at $\sqrt{s}=13 \mathrm{TeV}$ using single lepton events, J. High Energy Phys. 10 (2017) 019.

[4] M. Aaboud et al. (ATLAS Collaboration), Search for supersymmetry in final states with missing transverse momentum and multiple $b$-jets in proton-proton collisions at $\sqrt{s}=13 \mathrm{TeV}$ with the ATLAS detector, J. High Energy Phys. 06 (2018) 107.

[5] D. B. Kaplan, Flavor at SSC energies: A New mechanism for dynamically generated fermion masses, Nucl. Phys. B365, 259 (1991).

[6] N. Arkani-Hamed, M. A. Luty, and J. Terning, Composite quarks and leptons from dynamical supersymmetry breaking without messengers, Phys. Rev. D 58, 015004 (1998).

[7] M. A. Luty and J. Terning, Improved single sector supersymmetry breaking, Phys. Rev. D 62, 075006 (2000).

[8] T. Gherghetta and A. Pomarol, A Warped supersymmetric standard model, Nucl. Phys. B602, 3 (2001).

[9] M. Gabella, T. Gherghetta, and J. Giedt, A Gravity dual and LHC study of single-sector supersymmetry breaking, Phys. Rev. D 76, 055001 (2007).

[10] S. Franco and S. Kachru, Single-Sector supersymmetry breaking in supersymmetric QCD, Phys. Rev. D 81, 095020 (2010).

[11] N. Craig, R. Essig, S. Franco, S. Kachru, and G. Torroba, Dynamical supersymmetry breaking, with flavor, Phys. Rev. D 81, 075015 (2010).

[12] O. Aharony, L. Berdichevsky, M. Berkooz, Y. Hochberg, and D. Robles-Llana, Inverted sparticle hierarchies from natural particle hierarchies, Phys. Rev. D 81, 085006 (2010).

[13] G. Cacciapaglia, G. Marandella, and J. Terning, Dimensions of supersymmetric operators from AdS/CFT, J. High Energy Phys. 06 (2009) 027.

[14] E. Witten, Baryons in the 1/N Expansion, Nucl. Phys. B160, 57 (1979).

[15] Y. Buyukdag, T. Gherghetta, and A. Miller, Partially composite supersymmetry, Phys. Rev. D 99, 035046 (2019).

[16] R. Contino and A. Pomarol, Holography for fermions, J. High Energy Phys. 11 (2004) 058.

[17] B. Batell and T. Gherghetta, Holographic mixing quantified, Phys. Rev. D 76, 045017 (2007).
[18] J. M. Maldacena, The large N limit of superconformal field theories and supergravity, Int. J. Theor. Phys. 38, 1113 (1999); Adv. Theor. Math. Phys. 2, 231 (1998).

[19] L. Randall and R. Sundrum, A Large Mass Hierarchy from a Small Extra Dimension, Phys. Rev. Lett. 83, 3370 (1999).

[20] Y. Grossman and M. Neubert, Neutrino masses and mixings in nonfactorizable geometry, Phys. Lett. B 474, 361 (2000).

[21] T. Gherghetta and A. Pomarol, Bulk fields and supersymmetry in a slice of AdS, Nucl. Phys. B586, 141 (2000).

[22] Z. Chacko and E. Ponton, Bulk gauge fields in warped space and localized supersymmetry breaking, J. High Energy Phys. 11 (2003) 024.

[23] L. Randall and R. Sundrum, Out of this world supersymmetry breaking, Nucl. Phys. B557, 79 (1999).

[24] M. A. Luty and R. Sundrum, Hierarchy stabilization in warped supersymmetry, Phys. Rev. D 64, 065012 (2001).

[25] M. A. Luty, Weak Scale Supersymmetry without Weak Scale Supergravity, Phys. Rev. Lett. 89, 141801 (2002).

[26] T. Gherghetta and A. Pomarol, A Stuckelberg formalism for the gravitino from warped extra dimensions, Phys. Lett. B 536, 277 (2002).

[27] H. Itoh, N. Okada, and T. Yamashita, Low scale gravity mediation with warped extra dimension and collider phenomenology on the hidden sector, Phys. Rev. D 74, 055005 (2006).

[28] T. Gherghetta, B. von Harling, and N. Setzer, A natural little hierarchy for RS from accidental SUSY, J. High Energy Phys. 07 (2011) 011.

[29] J.E. Kim and H.P. Nilles, The $\mu$ problem and the strong CP-problem, Phys. Lett. 138B, 150 (1984).

[30] M. Bolz, A. Brandenburg, and W. Buchmuller, Thermal production of gravitinos, Nucl. Phys. B606, 518 (2001); Erratum B790, 336(E) (2008).

[31] J. Pradler and F. D. Steffen, Constraints on the reheating temperature in gravitino dark matter scenarios, Phys. Lett. B 648, 224 (2007).

[32] L. Bartoszek et al. (Mu2e Collaboration), Mu2e Technical Design Report, arXiv:1501.05241.

[33] V. Andreev et al. (ACME Collaboration), Improved limit on the electric dipole moment of the electron, Nature (London) 562, 355 (2018). 